\title{
Toward Modified Impact Inventory Tables to Facilitate Patient-Centered Value Assessment
}

\author{
R. Brett McQueen ${ }^{1,2}$ (1) $\cdot$ Julia F. Slejko ${ }^{1,2}$ \\ Accepted: 8 March 2021 / Published online: 24 March 2021 \\ (c) The Author(s), under exclusive licence to Springer Nature Switzerland AG 2021
}

\section{Introduction}

Value assessment is emerging as a key policy lever for pharmaceutical coverage and reimbursement decision making in the United States. Value frameworks largely rely on traditional value assessment methods such as comparative effectiveness research (CER), literature-based cost-effectiveness analyses (CEAs), and appraisal committees that deliberate the relative value of pharmaceutical interventions [1]. Main findings from value frameworks, in particular CEAs, can influence appraisal committee votes and policy recommendations, which may flow to coverage and reimbursement decisions [2].

Given value frameworks often take a population-level reimbursement perspective focused on clinical outcomes and payer costs, value assessments may omit the explicit evaluation of additional criteria important to patients [3, 4]. Specifically, there is a wide variety of value criteria that can be included in value assessment applications depending on decision context and perspective (e.g., additional measures of risk or uncertainty, equity, caregiver burden, stigma, etc.) [5-9]. However, health economists have not reached a consensus on how to measure and incorporate additional value criteria important to patients and broader society into value assessment applications [1, 4, 9]. Further, ISPOR's Special Task Force on Value Assessment Frameworks explicitly called for increased patient engagement and perspectives in value assessment applications [10].

R. Brett McQueen

Robert.mcqueen@cuanschutz.edu

1 Skaggs School of Pharmacy and Pharmaceutical Sciences, Department of Clinical Pharmacy, University of Colorado Anschutz Medical Campus, Mail Stop C238, 12850 E. Montview Blvd., Aurora, CO 80045, USA

2 University of Maryland School of Pharmacy, Baltimore, MA, USA
To address the call for increasing patient-centricity in value assessments [3, 4], we propose disease-specific modifications of a simple, yet powerful tool that has been recommended by the Second Panel on Cost-Effectiveness in Health and Medicine - the impact inventory table [11]. The impact inventory table provides researchers and consumers of value assessments a checklist of which value criteria are included to facilitate comparability with past and future assessments. While the impact inventory table includes the most important costs and societal effects, the criteria are largely generic across diseases and are often missing value criteria most important to patients.

Our proposed approach includes the following two-step process for modifying the generic impact inventory table to include additional patient-centric value criteria: (1) elicit and prioritize value criteria through patient engagement efforts; and (2) engage clinicians, value assessment researchers, and other stakeholders in a mapping exercise to determine the placement of prioritized and refined criteria to a modified disease-specific impact inventory table. Additionally, we provide a discussion on applications of modified disease-specific impact inventory tables using existing and potentially emerging novel value assessment methods. Our proposed approach refers to value assessment as defined by ISPOR's Special Task Force on Value Assessment where health care decisions can be made both at the population and individual level [10]. We define additional value criteria as disease- or treatment-specific attributes associated with health benefits, risks, and costs that are often not adequately captured by current value assessment applications. 


\section{Eliciting and Prioritizing Value Criteria to Inform Modified Impact Inventory Tables}

Step one relies on existing standards for patient engagement [4-6] that have been recently leveraged for use in value assessment. For example, the University of Maryland Patient-Driven Values in Healthcare Evaluation (PAVE) Center worked closely with patient stakeholders to identify a set of 42 disease-agnostic patient-informed 'value elements' categorized within 11 domains [7]. Ongoing research aims to tailor these disease-agnostic elements for use among diverse conditions and patient communities. Recently, these elements were tailored for the evaluation of treatments for chronic obstructive pulmonary disease (COPD), following an approach for value element prioritization and language refinement [12]. The aforementioned disease-agnostic elements were prioritized among a sample of individuals with COPD through an iterative process also including patient stakeholders. This process included guided activities conducted among individual participants who were asked to rank both the broad element domains and individual elements themselves. This iterative process resulted in refinement of a broad set of $40+$ elements into a list of six elements considered most important to COPD patients. Further engagement with patient experts and stakeholders led to the development of attribute and level phrasing for a stated preferences instrument, through which the prioritized elements may be further quantified in a future study. Prioritization and refinement within step one is a key milestone for informing the next step of mapping these criteria to disease-specific impact inventory tables.

\section{Mapping Patient-Centered Value Criteria to Modified Impact Inventory Tables}

Step two should engage clinicians, value assessment researchers, and other stakeholders in a mapping exercise to determine the placement of prioritized and refined criteria from step one in the disease-specific impact inventory table. Specifically, the top ranked domains and criteria (e.g., top 5-10) can be mapped to the impact inventory table by placing criteria within existing domains or expanding the number of domains that are disease-specific. For example, the current version of the impact inventory table stratifies domains by 'sector', including health in the formal and informal health care sectors, among other non-health-sector domains (e.g., education, housing etc.). Within health, the outcomes listed in the generic impact inventory table included longevity effects, health-related quality-of-life effects, and other health effects such as adverse events. Using the example provided by PAVE, we can expand the impact inventory table to include additional domains that are not already delineated in the generic table, such as 'Social Impact.' Other important domains may already be included in the impact inventory table but require expansion. For example, 'Short and Long-term Treatment Effects' are largely included in the formal health sector domain of the existing impact inventory table but those criteria within the broader domain may be updated with disease-specific criteria such as the PAVE example from COPD. As mentioned previously, it is critical that this step be a collaboration between multiple stakeholders. Examples of existing cross-disciplinary collaborations can inform the feasibility of step two, such as the International Consortium for Health Outcomes Measurement $[13,14]$.

\section{Applications of Disease-Specific Modified Impact Inventory Tables}

Modifying and expanding the generic impact inventory table may be useful in multiple applications for assessing the value of interventions. These applications may include but are not limited to traditional CER and CEA; augmented CEA (ACEA) to monetize additional value criteria [15]; multi-criteria decision analysis (MCDA), a useful technique to enable decision making by quantifying and weighting multiple criteria [16, 17]; and qualitative deliberation exercises such as discussions surrounding "other benefits and contextual considerations" that may or may not easily flow into a quantitative exercise [18]. Where modifications to the existing impact inventory table are minimal, traditional CER and CEA may be able to capture nearly all domains and criteria. In contrast, emerging novel value assessment methods such as ACEA and MCDA may be most useful for including domains and criteria that are not often included in the generic impact inventory table.

Eventually, modifying the impact inventory table for multiple disease-specific applications may result in a catalogue of tables that encompass the majority of morbidity and mortality. This concept is already gaining traction among health economics and outcomes researchers. For example, a recent paper by Kim and Neumann proposed modification of the generic impact inventory table to consider additional health and social consequences for the COVID-19 pandemic [19]. This timely and thoughtful paper was not exhaustive but set out key items for analysts to consider when evaluating policy responses to COVID-19.

Furthermore, as the field progresses with the use of novel value assessment methods, there are important gaps in existing 
field-based recommendations $[1,11]$ that threaten the consistency and standardization of value assessment applications. This is in part a reason why some value frameworks, such as the Institute for Clinical and Economic Review (ICER), restrict other benefits not captured by the quality-adjusted lifeyear (QALY) to qualitative deliberations [18]. Modifying the generic impact inventory table for disease-specific applications would also help inform these deliberative exercises by providing value assessors with a list of the most important domains and criteria to patients. In other words, regardless of whether these criteria may be included in a CER or CEA, they can still be discussed and included in value assessment applications in a way that is more patient-centric as compared to only generating these criteria from literature-based sources.

\section{Summary and Conclusions}

The Second Panel on Cost-Effectiveness in Health and Medicine developed a powerful yet simple tool to create consistency and standardization for CEAs in the United States. To consistently incorporate patient-centeredness in value assessment, we propose modifying the generic impact inventory table with disease-specific applications using a two-step process that engages patients and cross-disciplinary collaborations. Further disease-specific modifications would not prohibit the use of emerging value assessment methods such as ACEA or MCDA, instead they would allow and even promote value assessors to identify and include additional value criteria in their analyses regardless of the method. Additionally, researchers conducting cost-benefit analyses may also utilize expanded disease-specific impact inventory tables. In conclusion, disease-specific modifications to the generic impact inventory table are a feasible way to consistently and reliably provide a comprehensive list of patient-centered value criteria in both population- and individual-level value assessments.

\section{Declarations}

Funding This work was in part funded by a grant from the PhRMA Foundation's Value Assessment Initiative and the University of Colorado's Data Science to Patient Value Initiative. The views expressed in this manuscript were not contingent upon approval or influenced by the PhRMA Foundation or Data Science to Patient Value.

\section{Conflicts of interest None.}

Ethics approval Not applicable.

Consent to participate Not applicable.

Consent for publication Not applicable.

Availability of data and material Not applicable.

Code availability Not applicable.
Author contributions RBM drafted the first draft of this Editorial and JFS provided edits and contributions related to PAVE's research agenda.

\section{References}

1. Willke RJ, Neumann PJ, Garrison LP Jr, Ramsey SD. Review of recent US value frameworks-a health economics approach: an ISPOR Special Task Force Report [6]. Value Health. 2018;21(2):155-60.

2. CVS Health. Current and New Approaches to Making Drugs More Affordable 2018. https://cvshealth.com/sites/default/files/ cvs-health-current-and-new-approaches-to-making-drugs-moreaffordable.pdf. Accessed 20 Sept 2020.

3. Diaby V, Ali AA, Montero AJ. Value assessment frameworks in the united states: a call for patient engagement. Pharmacoecon Open. 2019;3(1):1-3.

4. Perfetto EM, Oehrlein EM, Boutin M, Reid S, Gascho E. Value to whom? The patient voice in the value discussion. Value Health. 2017;20(2):286-91.

5. Armstrong MJ, Mullins CD. Value assessment at the point of care: incorporating patient values throughout care delivery and a draft taxonomy of patient values. Value Health. 2017;20(2):292-5.

6. Avalere Health/FasterCures. Patient-Perspective Value Framework (PPVF) Washington, D.C.2016.

7. dosReis S, Butler B, Caicedo J, Kennedy A, Hong YD, Zhang C, et al. Stakeholder-engaged derivation of patient-informed value elements. Patient. 2020;13(5):611-21.

8. Slejko JF, Mattingly TJ 2nd, Mullins CD, Perfetto EM, dosReis S. Future of patients in healthcare evaluation: the patient-informed reference case. Value Health. 2019;22(5):545-8.

9. Lakdawalla DN, Doshi JA, Garrison LP Jr, Phelps CE, Basu A, Danzon PM. Defining elements of value in health care-a health economics approach: an Ispor special task force report [3]. Value Health. 2018;21(2):131-9.

10. Garrison LP Jr, Pauly MV, Willke RJ, Neumann PJ. An overview of value, perspective, and decision context-a health economics approach: an ispor special task force report [2]. Value Health. 2018;21(2):124-30.

11. Sanders GD, Neumann PJ, Basu A, Brock DW, Feeny D, Krahn $\mathrm{M}$, et al. Recommendations for conduct, methodological practices, and reporting of cost-effectiveness analyses: second panel on cost-effectiveness in health and medicine. JAMA. 2016;316(10):1093-103.

12. Slejko JF, Hong YD, Sullivan JL, Reed RM, dosReis S. Prioritization and refinement of patient-informed value elements as attributes for chronic obstructive pulmonary disease treatment preferences. Patient. 2021. https://doi.org/10.1007/s40271-021-00495-2.

13. International Consortium for Health Outcomes Measurement. 2021. https://www.ichom.org. Accessed 5 Feb 2021.

14. Nano J, Carinci F, Okunade O, Whittaker S, Walbaum M, Barnard-Kelly K, et al. A standard set of person-centred outcomes for diabetes mellitus: results of an international and unified approach. Diabet Med. 2020;37(12):2009-18.

15. Louis P, Garrison J, Zamora B, Li M, Towse A. Augmenting costeffectiveness analysis for uncertainty: the implications for value assessment—rationale and empirical support. J Manag Care Spec Pharm. 2020;26(4):400-6.

16. Marsh KD, Sculpher M, Caro JJ, Tervonen T. The use of MCDA in HTA: great potential, but more effort needed. Value in Health. 2018;21(4):394-7.

17. McQueen RB WM, Kalo Z, Longman R, Campbell JD. Complementing coverage and reimbursement decisions with multi-criteria 
decision analysis2019 2/27/2020. https://www.ajmc.com/contr ibutor/the-university-of-colorado-pharmaceutical-value/2019/ 05/complimenting-coverage-and-reimbursement-decisions-withmulticriteria-decision-analysis. Accessed 12 Sept 2020.

18. Institute for Clinical and Economic Review (ICER). Value Assessment Framework 2020. https://icer-review.org/wp-content/uploa
ds/2019/05/ICER_2020_2023_VAF_013120-4.pdf. Accessed 10 Sept 2020.

19. Kim DD, Neumann PJ. Analyzing the Cost Effectiveness of Policy Responses for COVID-19: The Importance of Capturing Social Consequences. Med Decis Mak. 2020;40(3):251-3. 